\title{
Mortality in retired coke oven plant workers
}

\author{
N Chau, J P Bertrand, J M Mur, A Figueredo, A Patris, J J Moulin, Q T Pham
}

Abstract

A previous study on 536 retired coke oven plant workers in Lorraine Collieries (France) reported an excess of deaths from lung cancer (standardised mortality ratio $(S M R)=251$ ) compared with the French male population. Occupational exposures during working life were retraced for each subject, but the number of deaths during the observation period (196382) was small, and smoking habits were known only for dead subjects. In 1988 , the cohort was re-examined (182 deaths occurred between 1963 and 1987) and smoking habits were determined for all the subjects. This study confirmed the excess of lung cancer ( $S M R=238$, $p<0.001)$. It showed an excess of mortality from all causes $(S M R=141, p<0.001)$, overall cancers $(S M R=133, p<0.05)$, and cardiovascular diseases $(S M R=133$, $p<0.05)$. A significant excess of deaths was found for subjects who worked near the ovens for all causes $(145, p<0.01)$, lung cancer $(\mathrm{SMR}=252, \quad \mathrm{p}<0.01), \quad$ colon cancer (SMR = 381, p < 0.05), and cardiovascular diseases $(S M R=155, p<0.05)$. A significant excess mortality was also found from all causes (176, $p<0.05)$ and stomach cancer (SMR $=538, p<0.01)$ in subjects who worked in byproducts, from lung cancer $(S M R=433$, $p<0.001$ ) in those in the workshops, and from cirrhosis of the liver and alcoholism (SMR $=360, \mathrm{p}<0.01$ ) in those underground; but, due to small numbers, these figures were not robust. An excess of mortality from all

INSERM U 115 "Santé au Travail et Santé Publique: Méthodes et Applications", Faculté de Médecine, $\mathrm{BP} 184,54505$ Vandoeuvre-lès-Nancy Cedex, France N Chau, J M Mur, A Patris, Q T Pham

Service de Médecine du Travail, Houillères du Bassin de Lorraine, Freyming-Merlebach

J P Bertrand, A Figueredo

Institut National de Recherche et de Sécurité (INRS), Vandoeuvre-lès-Nancy

J M Mur, J J Moulin

Laboratoire d'Informatique Médicale, Faculté de Médecine, Vandoeuvre-lès-Nancy

A Patris causes $(S M R=163, p<0.01)$, lung cancer $(\mathrm{SMR}=228, p<0.05)$, and cardiovascular diseases $(S M R=179, p<0.01)$ was shown also for non-exposed or slightly exposed subjects. The fact that, on the whole, mortality of various exposed groups was similar to that of non-exposed or slightly exposed workers may be explained in part by the selection at hiring and the healthy worker effect. As an increased risk of lung cancer was noted among subjects who worked in the old generations of plant compared with the other workers (although the relative risk was not significant) it is concluded that the role of occupational hazards could not be excluded.

(British Journal of Industrial Medicine 1993;50:127-135)

The effect of occupational hazards in coke oven plants (notably of polycyclic aromatic hydrocarbon from the ovens, coal dusts, tar, etc) on mortality has been extensively investigated. ${ }^{1-30}$ A previous study carried out in 1983 on 536 retired workers from the two coke oven plants of Houillères du Bassin de Lorraine (Lorraine Collieries) reported a significant excess of mortality from lung cancer $(\mathrm{SMR}=251, \mathrm{p}<0.01)$ with reference to the French male population. ${ }^{1}$ The small number of deaths found during the observation period (1963-82) did not allow a full analysis of the effect of occupational exposures. Also, smoking habits were known only for dead subjects. In 1988, the cohort was re-examined, and smoking habits were determined for all the cohort members. A question of interest was whether there was an increased risk, notably for lung cancer, in the old generations of plant. In this study we analysed the effect of occupational hazards on subjects who worked on or near the ovens, but also on those in other exposed workplaces-namely, byproducts, workshops, and underground. All causes of death were studied, in particular various cancers and cardiovascular diseases.

Subjects who were still alive on 1 January 1988 (354 subjects) were asked to attend a medical examination at the firm's occupational health centre for a study on morbidity ${ }^{31}$ and on lung function. ${ }^{32}$ 
Materials and methods

In the Lorraine Collieries, coke is produced in two plants: plant A started production in $1911\left(A_{\text {old }}\right)$ and plant $B$ in 1955. The occupational hazards in plant $A$ were reduced since the opening of $A_{\text {new }}$ in 1963. The occupational exposure in $B$ is considered as intermediate between $A_{\text {old }}$ and $A_{\text {new }}$. The study population consisted of all the male manual workers from the two plants who had retired between 1 January 1963 and 31 December 1982; a total of 536 subjects (the date of entry in the study was that of retirement). They were born between 1902 and 1935. Seventy subjects $(13.1 \%)$ worked in the old generations of plant A. Executives were excluded from the study. The observation period ranged from 1 January 1963 to 31 December 1987 (25 years). During this period, 182 deaths occurred $(34 \cdot 0 \%)$.

Causes of death have been established from the medical records kept by hospitals, occupational physicians, general practitioners, and physicians from the health services. In this study, only the main cause of death was considered; it was coded according to the 9th revision of the International Classification of Diseases. ${ }^{33}$ These records allowed us also to define the smoking habit for each subject. Information gathered from the subject himself and his family enabled us to complete and confirm data from medical records. Because of lack of precision and small numbers, we did not differentiate between the various categories of smokers or distinguish between smokers and ex-smokers.

\section{OCCUPATIONAL EXPOSURES}

For each subject, the job history was retraced to define the occupational hazards during his working life. The job history was broken down into a succession of occupations, for each of which were defined the date of the beginning and the end of each occupation, the job, the workplace, and the occupational nuisances. The analysis of the jobs held during a career showed that almost all of the subjects had one dominant job. This led us to define six types of homogeneous exposure with each subject being classified in the exposure group that corresponded to the longest duration of exposure when this period equalled or exceeded five years. The six were: ${ }^{132}$

(1) Constant presence on the coke ovens (noted ovens): support setter, shield setter, oven regulator, door cleaner, column cleaner, and tar cleaning tube operator.

(2) Activity near the ovens and intermittent presence on the ovens (noted $\mathrm{N}$ ovens): pusher machine operator, stamper, switch operator, coke car, maintenance personnel on the ovens, fitter.

(3) Exposure in repair and maintenance workshops (noted workshops).

(4) Exposure to byproducts (noted byproducts) in the oven gas treatment sector: benzol, phenol, sulphates, pitch, tars, etc.

(5) Underground work in coalmines (noted underground).

(6) Non- or slightly exposed (noted non-exposed): administrative personnel, time keeper, maintenance personnel who did not come into direct contact with the ovens, coal mixing work, porter, etc.

\section{STATISTICAL METHODS USED}

The statistical analysis consisted mainly in comparing each exposed group with the non-exposed group. Comparison of the subjects' age was made by analysis of variance and that of smoking with $\chi^{2}$ independence test or Fisher's exact probability test. ${ }^{34} 35$ The mortality analysis was made using the standardised mortality ratio (SMR) $(\%)^{36-38}$ with the French male population as standard population. ${ }^{39}$ The SMRs were calculated with five year age groups, for each year of the observation period. Comparison of mortality between the two plants was made with the homogeneity $\chi^{2}$ test of SMRs. ${ }^{36}{ }^{38}$ To assess the risk of an exposed group $v$ the non-exposed one we calculated the relative risk (ratio of SMRs) when these SMRs were significant. To estimate the effect of the old generations of plant $A$ on lung cancer the relative risk was also calculated, differentiating two categories of workers: subjects who worked only in the plant $A_{\text {new }}$ and those who worked in $A_{\text {old }}$. All the statistical tests were carried out with a first order risk of 0.05 excluding the non-responses. The different statistical analyses were made with the Logist program. ${ }^{40}$

\section{Results}

The study population consisted of 536 retirees. During the study period 182 deaths occurred $(34.0 \%)$. Table 1 presents the characteristics of the occupational exposure groups. The total duration of exposure ranged from 18 to 24 years. The nonexposed subjects had an average total duration of exposure of 1.2 years. Smoking for each exposed group was similar to that of the non-exposed group. The mean age at death was $64 \cdot 8$ years. The subjects of the underground group had an age at death lower than that of the other groups and they entered the study younger.

The main causes of death were cardiovascular diseases $(35 \cdot 2 \%)$ and cancers $(33.0 \%)$. Lung cancer was the cause in $13.7 \%$ of the cases, cirrhosis of the liver and alcoholism in $7 \cdot 1 \%$, and respiratory diseases in $4.9 \%$. Each of the other causes accounted for less than $3 \%$ of cases. The frequency of unknown causes $(11.0 \%$; table 2$)$ was close to the figure of the national statistics.

For all the cohort, a significant excess mortality (in reference to the French male population) was found for all causes $(S M R=141, p<0.01)$, cardio- 
Table 1 Characteristics of occupational exposure groups

\begin{tabular}{|c|c|c|c|c|c|c|c|}
\hline & \multirow[b]{2}{*}{ Total } & \multicolumn{6}{|l|}{ Group } \\
\hline & & Non-exposed & Ovens & Novens & Workshops & Byproducts & Underground \\
\hline \multicolumn{8}{|c|}{ All the cohort } \\
\hline No of subjects & 536 & 126 & 65 & 165 & 61 & 42 & 77 \\
\hline $\begin{array}{l}\text { Smoking (\% of subjects): } \\
\text { Non-smokers } \\
\text { Smokers } \\
\text { Unknown }\end{array}$ & $\begin{array}{l}22 \cdot 6 \\
64 \cdot 2 \\
13 \cdot 2\end{array}$ & $\begin{array}{l}22 \cdot 2 \\
66 \cdot 7 \\
11 \cdot 1\end{array}$ & $\begin{array}{r}24 \cdot 6 \\
66 \cdot 2 \\
9 \cdot 2\end{array}$ & $\begin{array}{l}22 \cdot 4 \\
64 \cdot 9 \\
12 \cdot 7\end{array}$ & $\begin{array}{r}24 \cdot 6 \\
67 \cdot 2 \\
8 \cdot 2\end{array}$ & $\begin{array}{l}26 \cdot 2 \\
45 \cdot 3 \\
28 \cdot 6\end{array}$ & $\begin{array}{l}18 \cdot 2 \\
65 \cdot 0 \\
16 \cdot 9\end{array}$ \\
\hline $\begin{array}{l}\text { Duration of different types of } \\
\text { Ovens } \\
\text { Novens } \\
\text { Workshops } \\
\text { Byproducts } \\
\text { Underground }\end{array}$ & $\begin{array}{c}\text { f exposure }(\mathrm{m} \\
1 \cdot 8(5 \cdot 0) \\
5 \cdot 2(8 \cdot 7) \\
3 \cdot 7(8 \cdot 1) \\
2 \cdot 1(7 \cdot 2) \\
4 \cdot 2(7 \cdot 4)\end{array}$ & $\begin{array}{l}\text { (SD) (y)): } \\
0 \cdot 1(0 \cdot 5) \\
0 \cdot 2(0 \cdot 7) \\
0 \cdot 2(0 \cdot 7) \\
0 \cdot 1(0 \cdot 7) \\
0 \cdot 6(1 \cdot 2)\end{array}$ & $\begin{array}{r}14 \cdot 0(5 \cdot 9) \\
2 \cdot 7(5 \cdot 6) \\
2 \cdot 2(5 \cdot 9) \\
0 \cdot 1(0 \cdot 7) \\
3 \cdot 7(7 \cdot 2)\end{array}$ & $\begin{array}{r}0.3(0.9) \\
15 \cdot 6(8 \cdot 6) \\
3.0(6 \cdot 7) \\
1 \cdot 0(4 \cdot 4) \\
3 \cdot 2(5 \cdot 8)\end{array}$ & $\begin{array}{l}\overline{0} \\
0.1(0 \cdot 6) \\
20 \cdot 7(8 \cdot 8) \\
0.4(2 \cdot 1) \\
0.7(1 \cdot 8)\end{array}$ & $\begin{array}{l}0.07(0 \cdot 4) \\
0 \cdot 8(3 \cdot 2) \\
22 \cdot 1(11 \cdot 8) \\
1 \cdot 0(2 \cdot 1)\end{array}$ & $\begin{array}{l}0 \cdot 1(0 \cdot 6) \\
0 \cdot 3(1 \cdot 1) \\
0 \cdot 3(1 \cdot 0) \\
0 \cdot 02(0 \cdot 1) \\
17 \cdot 4(6 \cdot 8)\end{array}$ \\
\hline \multicolumn{8}{|c|}{ Dead subjects } \\
\hline No of deaths & 182 & 54 & 17 & 56 & 15 & 18 & 22 \\
\hline Age at death (mean (SD) (y)) & $64 \cdot 8(7 \cdot 3)$ & $65 \cdot 2(7 \cdot 1)$ & $64 \cdot 1(5 \cdot 8)$ & $66 \cdot 4(6 \cdot 7)$ & $64 \cdot 7(7 \cdot 2)$ & $64 \cdot 3(8 \cdot 1)$ & $60 \cdot 7(8 \cdot 6)^{\star}$ \\
\hline
\end{tabular}

${ }^{\star} \mathrm{p}<0.05$, comparison of means and homogeneity test between each exposed group and the non-exposed group (all the smokers were grouped together; unknown responses were excluded).

vascular diseases $(S M R=133, p<0.05)$, overall cancers $(S M R=133, p<0.05)$, and especially lung cancer $(S M R=238, p<0.01)$. Other cancers (upper respiratory and alimentary tract, oesophagus, stomach, bladder, etc) were under-represented (table 3).

An excess of overall mortality was seen for all groups but it was significant only for subjects who worked near the ovens (SMR $=145, p<0.01)$, in byproducts $(S M R=176, p<0.05)$, and also for non-exposed workers $(S M R=163, p<0.001)$. Mortality from overall cancer was also higher than expected for all groups but the excess was not significant. The SMR for lung cancer was above 1.00 for all groups except the underground group, but it was significant only for workers near the ovens (252, $\mathrm{p}<0.01$ ), in the workshops $(433, \mathrm{p}<0.001)$, and non-exposed $(228, p<0.05)$. Compared with nonexposed subjects, those near the ovens and those in the workshops had a relative risk for lung cancer of

Table 2 Distribution of causes of death (\%)

\begin{tabular}{|c|c|c|c|c|c|c|c|}
\hline \multirow[b]{2}{*}{ Cause of death (ICD-9) } & \multirow[b]{2}{*}{ Total } & \multicolumn{5}{|l|}{ Group } & \multirow[b]{2}{*}{ Underground } \\
\hline & & Non-exposed & Ovens & Novens & Workshops & Byproducts & \\
\hline No of subjects & 182 & 54 & 17 & 56 & 15 & 18 & 22 \\
\hline $\begin{array}{l}\text { Cancer, all sites (140-239) } \\
\text { Lung (162) } \\
\text { Upper respiratory tract, } \\
\text { alimentary tract }\end{array}$ & $\begin{array}{l}33 \cdot 0 \\
13 \cdot 7\end{array}$ & $\begin{array}{l}24 \cdot 1 \\
11 \cdot 1\end{array}$ & $\begin{array}{l}47 \cdot 1 \\
11 \cdot 8\end{array}$ & $\begin{array}{l}32 \cdot 1 \\
14 \cdot 3\end{array}$ & $\begin{array}{l}66 \cdot 7 \\
40 \cdot 0\end{array}$ & $\begin{array}{l}27 \cdot 8 \\
11 \cdot 1\end{array}$ & $\begin{array}{r}27 \cdot 3 \\
4 \cdot 5\end{array}$ \\
\hline $\begin{array}{l}(140-149,161) \\
\text { Oesophagus }(150)\end{array}$ & $\begin{array}{l}2 \cdot 2 \\
1 \cdot 6\end{array}$ & $\begin{array}{r}1 \cdot 8 \\
3 \cdot 7\end{array}$ & $\begin{array}{l}5 \cdot 9 \\
5.9\end{array}$ & $-^{1.8}$ & $-^{6.7}$ & - & 二 \\
\hline Stomach (151) & $2 \cdot 2$ & - & 5.9 & 一 & - & $11 \cdot 1$ & $4 \cdot 5$ \\
\hline Bladder (188) & 0.5 & - & - & $1 \cdot 8$ & - & - & \\
\hline $\begin{array}{l}\text { Colon (153) } \\
\text { Rectum (154; }\end{array}$ & $2 \cdot 2$ & 二 & 二 & 5.4 & - & - & 4.5 \\
\hline $\begin{array}{l}\text { Rectum (154) } \\
\text { Haemopathies (200-209) }\end{array}$ & $\begin{array}{l}0.5 \\
1.1\end{array}$ & 二 & -5.9 & $-^{1.8}$ & ${ }_{6.7}$ & - & 二 \\
\hline Other sites & $8 \cdot 8$ & $7 \cdot 4$ & $11 \cdot 8$ & $7 \cdot 1$ & $13 \cdot 3$ & $5 \cdot 6$ & $13 \cdot 6$ \\
\hline $\begin{array}{l}\text { Infectious diseases (000-139) } \\
\text { Cardiovascular diseases }\end{array}$ & $1 \cdot 6$ & - & - & $1 \cdot 8$ & - & $5 \cdot 6$ & - \\
\hline $\begin{array}{l}(390-459) \\
\text { Respiratory diseases }\end{array}$ & $35 \cdot 2$ & $42 \cdot 6$ & $35 \cdot 3$ & $39 \cdot 3$ & $13 \cdot 3$ & $22 \cdot 2$ & $31 \cdot 8$ \\
\hline $\begin{array}{l}(460-519) \\
\text { Cirrhosis of liver }\end{array}$ & $4 \cdot 9$ & $7 \cdot 4$ & - & 8.9 & - & - & - \\
\hline $\begin{array}{l}\text { Cirrhosis of liver, } \\
\text { alcoholism }(571.0-3,5)\end{array}$ & $7 \cdot 1$ & $3 \cdot 7$ & $11 \cdot 8$ & $3 \cdot 6$ & $13 \cdot 3$ & $5 \cdot 6$ & $18 \cdot 2$ \\
\hline $\begin{array}{l}\text { Other digestive tract diseases } \\
\text { Accidents, other violence }\end{array}$ & 1.7 & $1 \cdot 8$ & - & $1 \cdot 8$ & - & $5 \cdot 6$ & \\
\hline (E800-999) & $2 \cdot 8$ & $3 \cdot 7$ & - & $1 \cdot 8$ & - & - & $9 \cdot 1$ \\
\hline $\begin{array}{l}\text { Other causes } \\
\text { Undefined causes }\end{array}$ & 1.6 & 3.7 & - & - & - & $5 \cdot 6$ & \\
\hline $\begin{array}{l}\text { Undefined causes } \\
\text { Unknown causes }\end{array}$ & $\begin{array}{r}1 \cdot 1 \\
11 \cdot 0\end{array}$ & $\overline{11} \cdot 1$ & $-^{5.9}$ & $\overline{10} \cdot 7$ & $-_{6.7}$ & $\overline{27.8}$ & $\begin{array}{l}4 \cdot 5 \\
9 \cdot 1\end{array}$ \\
\hline All causes & $100 \cdot 0$ & $100 \cdot 0$ & $100 \cdot 0$ & $100 \cdot 0$ & $100 \cdot 0$ & $100 \cdot 0$ & $100 \cdot 0$ \\
\hline
\end{tabular}

${ }^{\star} \mathrm{p}<0.01$, comparison of frequencies between each exposed group and the non-exposed group. 
Table 3 Mortality according to occupational exposure

\begin{tabular}{|c|c|c|c|c|c|c|c|c|c|c|c|c|c|c|}
\hline \multirow[b]{3}{*}{ Cause of death (ICD-9) } & \multirow{2}{*}{\multicolumn{2}{|c|}{ Total }} & \multicolumn{12}{|c|}{ Group } \\
\hline & & & \multicolumn{2}{|c|}{ Non-exposed } & \multicolumn{2}{|c|}{ Ovens } & \multicolumn{2}{|c|}{ Novens } & \multicolumn{2}{|c|}{ Workshops } & \multicolumn{2}{|c|}{ Byproducts } & \multicolumn{2}{|c|}{ Underground } \\
\hline & $O$ & $S M R$ & $O$ & $S M R$ & $O$ & $S M R$ & $O$ & $S M R$ & $O$ & $S M R$ & $O$ & $S M R$ & $O$ & SMR \\
\hline All causes & 182 & 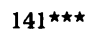 & 54 & $163^{\star \star \star}$ & 17 & 132 & 56 & $145^{\star \star}$ & 15 & 87 & 18 & $176^{\star}$ & 22 & 130 \\
\hline $\begin{array}{l}\text { Cancer, all sites (140-239) } \\
\text { Lung (162) } \\
\text { Upper respiratory and } \\
\text { alimentary tract }\end{array}$ & $\begin{array}{l}60 \\
25\end{array}$ & $\begin{array}{l}133^{\star} \\
238^{\star \star \star}\end{array}$ & $\begin{array}{r}13 \\
6\end{array}$ & $\begin{array}{l}114 \\
228^{\star}\end{array}$ & $\begin{array}{l}8 \\
2\end{array}$ & $\begin{array}{l}168 \\
175\end{array}$ & $\begin{array}{r}18 \\
8\end{array}$ & $\begin{array}{l}133 \\
252^{\star \star}(\mathrm{a})\end{array}$ & $\begin{array}{r}10 \\
6\end{array}$ & $\begin{array}{l}166 \\
433^{\star \star \star}(b)\end{array}$ & $\begin{array}{l}5 \\
2\end{array}$ & $\begin{array}{l}138 \\
237\end{array}$ & & $\begin{array}{r}103 \\
74\end{array}$ \\
\hline $\begin{array}{l}(140-149,161) \\
\text { Oesophagus }(150) \\
\text { Stomach }(151) \\
\text { Bladder (188) } \\
\text { Colon }(153) \\
\text { Rectum }(154)\end{array}$ & $\begin{array}{l}4 \\
3 \\
4 \\
1 \\
4 \\
1\end{array}$ & $\begin{array}{r}64 \\
90 \\
91 \\
70 \\
151 \\
71\end{array}$ & $\begin{array}{l}1 \\
2 \\
- \\
- \\
-\end{array}$ & $\begin{array}{l}66 \\
242 \\
(1 \cdot 19) \\
(0 \cdot 37) \\
(0 \cdot 68) \\
(0 \cdot 37)\end{array}$ & $\begin{array}{l}1 \\
1 \\
1 \\
= \\
-\end{array}$ & $\begin{array}{l}146 \\
283 \\
227 \\
(0 \cdot 14) \\
(0 \cdot 27) \\
(0 \cdot 14)\end{array}$ & $\begin{array}{l}1 \\
\overline{1} \\
1 \\
3 \\
1\end{array}$ & $\begin{array}{l}53 \\
(1 \cdot 01) \\
(1 \cdot 31) \\
237 \\
381 \star \\
238\end{array}$ & $\begin{array}{l}\frac{1}{-} \\
- \\
-\end{array}$ & $\begin{array}{l}126 \\
(0.43) \\
(0 \cdot 59) \\
(0 \cdot 19) \\
(0.36) \\
(0 \cdot 19)\end{array}$ & $\begin{array}{l}- \\
\frac{2}{-} \\
-\end{array}$ & $\begin{array}{l}(0 \cdot 49) \\
(0 \cdot 27) \\
538^{\star \star} \\
(0 \cdot 11) \\
(0 \cdot 21) \\
(0 \cdot 11)\end{array}$ & $\begin{array}{l}- \\
\frac{1}{1} \\
-\end{array}$ & $\begin{array}{l}(0 \cdot 85) \\
(0 \cdot 44) \\
197 \\
(0 \cdot 18) \\
301 \\
(0 \cdot 18)\end{array}$ \\
\hline \multirow{4}{*}{$\begin{array}{l}\text { Cardiovascular diseases } \\
(390-459) \\
\text { Respiratory diseases } \\
(460-519) \\
\text { Cirrhosis of liver, } \\
\text { alcoholism }(571 \cdot 0-3,5) \\
\text { Accidents, other violence } \\
\text { (E800-999) }\end{array}$} & 64 & $133^{\star}$ & 23 & $179 \star \star$ & 6 & 123 & 22 & $155^{\star}$ & 2 & 30 & 4 & 100 & 7 & 124 \\
\hline & 6 & 68 & 4 & 171 & - & $(0 \cdot 89)$ & 2 & 77 & - & $(1 \cdot 22)$ & - & $(0 \cdot 73)$ & 一 & $(1.03)$ \\
\hline & 13 & 137 & 2 & 82 & 2 & 192 & 2 & 70 & 2 & 160 & 1 & 124 & 4 & $360 \star \star$ \\
\hline & 5 & 54 & 2 & 86 & - & $(0.96)$ & 1 & 36 & - & $(1 \cdot 20)$ & - & $(0 \cdot 74)$ & 2 & 153 \\
\hline
\end{tabular}

${ }^{\star} \mathrm{p}<0.05 ;{ }^{\star \star} \mathrm{p}<0.01 ;{ }^{\star \star \star} \mathrm{p}<0.001$.

$\mathrm{O}=$ Number of observed deaths. When $\mathrm{O}$ equals zero, the number of expected deaths is given in parentheses.

(a) Relative risk compared with the non-exposed group $(R R=$ ratio of $S M R s)=1 \cdot 11, N S$; (b) $R R=1 \cdot 90, N S$.

$1 \cdot 11$ (NS) and 1.90 (NS) respectively. This study showed a significant $S M R$ for stomach cancer for men in byproducts $(538, p<0.01)$, for colon cancer for those near the ovens $(381, \mathrm{p}<0.05)$, for cardiovascular diseases for non-exposed workers and those near the ovens $(179, \mathrm{p}<0.01$ and 155, p $<0.05$ respectively), and for cirrhosis of the liver and alcoholism for men working underground (360, p < 0.01) (table 3).

Tables 4 and 5 show the effect of smoking for each exposure group. Because of small numbers, workers on and those near the ovens were grouped together for each type of cancer. Firstly, although the frequency of subjects with unknown smoking habits was low $(13.2 \%)$, it was easier to determine the smoking habits for survivors than for dead subjects $(7.9 \% v 23.6 \%, \mathrm{p}<0.001)$. Thus mortality of smokers and non-smokers may be underestimated. For all causes, although the smokers had a significant SMR $(p<0.01)$ and the non-smokers a nonsignificant SMR, their SMRs were close (130 $v 127$ respectively). A significant excess of all cancers was found only for smokers (SMR $=150, p<0.01$ ); this was due to the men working on the ovens

Table 4 Mortality according to occupational exposure and smoking

\begin{tabular}{|c|c|c|c|c|c|c|c|c|c|c|c|c|c|c|}
\hline \multirow[b]{3}{*}{ Cause of death (ICD-9) } & \multirow{2}{*}{\multicolumn{2}{|c|}{ Total }} & \multicolumn{12}{|c|}{ Group } \\
\hline & & & \multicolumn{2}{|c|}{ Non-exposed } & \multicolumn{2}{|c|}{ Ovens } & \multicolumn{2}{|c|}{$N$ ovens } & \multicolumn{2}{|c|}{ Workshops } & \multicolumn{2}{|c|}{ Byproducts } & \multicolumn{2}{|c|}{ Underground } \\
\hline & $O$ & $S M R$ & $O$ & $S M R$ & $O$ & $S M R$ & $O$ & $S M R$ & $O$ & $S M R$ & $O$ & $S M R$ & $O$ & $S M R$ \\
\hline \multicolumn{15}{|l|}{ All causes: } \\
\hline Non-smokers & 40 & 127 & 11 & 136 & 4 & 120 & 12 & 140 & 6 & 128 & 4 & 129 & 3 & 81 \\
\hline Smokers & 99 & $130^{\star \star}$ & 33 & $155^{\star}$ & 12 & 151 & 31 & 133 & 7 & 68 & 5 & 119 & 11 & 116 \\
\hline \multicolumn{15}{|c|}{ Cancer, all sites (140-239): } \\
\hline Non-smokers & 11 & 99 & 1 & 35 & 1 & 82 & 2 & 65 & 3 & 193 & 3 & 275 (a) & 1 & 77 \\
\hline Smokers & 41 & $150 \star \star$ & 10 & 137 & 7 & $239^{\star}$ & $1 \overline{2}$ & 141 & 6 & 164 & 2 & 128 & 4 & 118 \\
\hline Unknown & 8 & 118 & 2 & 164 & - & $(0 \cdot 62)$ & 4 & 199 & 1 & 126 & - & $(0.97)$ & 1 & 87 \\
\hline \multicolumn{15}{|c|}{ Cardiovascular diseases (390-459): } \\
\hline Non-smokers & 20 & $166^{\star}$ & 8 & $235^{\star}$ & 2 & 172 & 7 & $224^{\star}$ & 2 & 119 & - & $(1 \cdot 28)$ & 1 & 71 \\
\hline Smokers & 35 & 121 & 14 & $169 \star$ & 3 & 102 & 12 & 134 & - & $(3.99)$ & 2 & 114 & 4 & 131 \\
\hline Unknown & 9 & 127 & 1 & 85 & 1 & 132 & 3 & 145 & - & $(0.96)$ & 2 & 205 & 2 & 174 \\
\hline Non-smokers & 2 & 84 & - & $(0.67)$ & 1 & 398 & - & $(0 \cdot 64)$ & 1 & 349 & - & $(0 \cdot 25)$ & - & $(0 \cdot 27)$ \\
\hline Smokers & 9 & 151 & 2 & 129 & 1 & 160 & 2 & 104 & 1 & 124 & 1 & 260 & 2 & 298 \\
\hline Unknown & 2 & 169 & - & $(0 \cdot 21)$ & - & $(0 \cdot 16)$ & - & $(0 \cdot 32)$ & - & $(0 \cdot 15)$ & - & $(0 \cdot 17)$ & 2 & $1193^{\star \star \star}$ \\
\hline
\end{tabular}

${ }^{\star} \mathrm{p}<0.05 ;{ }^{\star \star} \mathrm{p}<0.01 ;{ }^{\star \star \star} \mathrm{p}<0.001 ;$ (a) $\mathrm{p}<0.09$.

$\mathrm{O}=$ Number of observed deaths. When $\mathrm{O}$ equals zero, the number of expected deaths is given in parentheses. 
Table 5 Mortality from some cancers according to occupational exposure and smoking

\begin{tabular}{|c|c|c|c|c|c|c|c|c|c|c|c|c|c|}
\hline \multirow[b]{3}{*}{ Cause of death (ICD-9) } & \multirow{2}{*}{\multicolumn{2}{|c|}{ Total }} & \multicolumn{11}{|c|}{ Group } \\
\hline & & & \multicolumn{2}{|c|}{ Non-exposed } & \multirow{2}{*}{ Ovens } & \multirow{2}{*}{+} & \multirow{2}{*}{$\frac{N \text { ovens }}{S M R}$} & \multicolumn{2}{|c|}{ Workshops } & \multicolumn{2}{|c|}{ Byproducts } & \multicolumn{2}{|c|}{ Underground } \\
\hline & $O$ & $S M R$ & $O$ & $S M R$ & & & & $O$ & $S M R$ & $O$ & $S M R$ & $O$ & $S M R$ \\
\hline $\begin{array}{l}\text { Lung cancer (162): } \\
\text { Non-smokers } \\
\text { Smokers } \\
\text { Unknown }\end{array}$ & $\begin{array}{r}1 \\
20 \\
4\end{array}$ & $\begin{array}{l}39 \\
310^{\star \star \star} \\
265^{\star}\end{array}$ & $\begin{array}{l}\overline{5} \\
1\end{array}$ & $\begin{array}{l}(0 \cdot 66) \\
297^{\star} \\
360\end{array}$ & $\begin{array}{l}\overline{8} \\
2\end{array}$ & & $\begin{array}{l}(1 \cdot 01) \\
294^{\star \star}(a) \\
345\end{array}$ & $\begin{array}{l}1 \\
5 \\
1\end{array}$ & $\begin{array}{l}166 \\
581^{\star \star \star}(\mathrm{b}) \\
560\end{array}$ & $\begin{array}{l}0 \\
1 \\
-\end{array}$ & $\begin{array}{c}0 \\
268 \\
(0 \cdot 22)\end{array}$ & 二 & $\begin{array}{l}(0.30) \\
(0.50) \\
(0.25)\end{array}$ \\
\hline $\begin{array}{l}\text { Upper respiratory and alin } \\
\text { Non-smokers } \\
\text { Smokers } \\
\text { Unknown }\end{array}$ & $\frac{1}{3}$ & $\begin{array}{l}\text { tract can } \\
(1.51) \\
78 \\
114\end{array}$ & $\frac{\operatorname{er}(1}{1}$ & $\begin{array}{l}0-149,161): \\
(0 \cdot 39) \\
(0 \cdot 97) \\
620^{\star}\end{array}$ & $\frac{-}{2}$ & & $\begin{array}{l}(0 \cdot 61) \\
122 \\
(0 \cdot 35)\end{array}$ & $\frac{-}{1}$ & $\begin{array}{l}(0 \cdot 19) \\
196 \\
(0 \cdot 22)\end{array}$ & 二 & $\begin{array}{l}(0 \cdot 14) \\
(0 \cdot 23) \\
(0 \cdot 13)\end{array}$ & 二 & $\begin{array}{l}(0.18) \\
(0.53) \\
(0.15)\end{array}$ \\
\hline $\begin{array}{l}\text { Oesophageal cancer (150): } \\
\text { Non-smokers } \\
\text { Smokers } \\
\text { Unknown }\end{array}$ & $\frac{-}{-}$ & $\begin{array}{l}(0 \cdot 81) \\
148 \\
(0 \cdot 48)\end{array}$ & $\frac{1}{1}$ & $\begin{array}{l}(0 \cdot 21) \\
381 \\
(0 \cdot 09)\end{array}$ & $\frac{1}{1}$ & & $\begin{array}{l}(0 \cdot 32) \\
119 \\
(0 \cdot 19)\end{array}$ & E & $\begin{array}{l}(0 \cdot 11) \\
(0 \cdot 27) \\
(0 \cdot 05)\end{array}$ & 二 & $\begin{array}{l}(0.08) \\
(0.12) \\
(0.07)\end{array}$ & 二 & $\begin{array}{l}(0.09) \\
(0.26) \\
(0.08)\end{array}$ \\
\hline $\begin{array}{l}\text { Stomach cancer (151): } \\
\text { Non-smokers } \\
\text { Smokers } \\
\text { Unknown }\end{array}$ & $\frac{3}{1}$ & $\begin{array}{l}268 \\
(2 \cdot 64) \\
152\end{array}$ & 二 & $\begin{array}{l}(0.32) \\
(0.75) \\
(0.12)\end{array}$ & $\frac{1}{-}$ & & $\begin{array}{l}250 \\
(1.09) \\
(0 \cdot 26)\end{array}$ & E & $\begin{array}{l}(0 \cdot 15) \\
(0 \cdot 36) \\
(0 \cdot 09)\end{array}$ & $\frac{2}{-}$ & $\begin{array}{l}1676^{\star \star \star} \\
(0 \cdot 16) \\
(0.09)\end{array}$ & $\bar{z}$ & $\begin{array}{l}(0 \cdot 13) \\
(0 \cdot 28) \\
969 \star \star\end{array}$ \\
\hline $\begin{array}{l}\text { Colon and rectum cancer } \\
\text { Non-smokers } \\
\text { Smokers } \\
\text { Unknown }\end{array}$ & $\frac{3-15}{4}$ & $\begin{array}{l}\text { (i.02) } \\
168 \\
152\end{array}$ & 二 & $\begin{array}{l}(0 \cdot 26) \\
(0 \cdot 67) \\
(0 \cdot 12)\end{array}$ & $\frac{-3}{-}$ & & $\begin{array}{l}(0 \cdot 38) \\
306^{\star} \\
385\end{array}$ & - & $\begin{array}{l}(0.15) \\
(0.32) \\
(0.08)\end{array}$ & - & $\begin{array}{l}(0.11) \\
(0.15) \\
(0.09)\end{array}$ & $\frac{1}{-}$ & $\begin{array}{l}(0 \cdot 12) \\
369 \\
(0 \cdot 11)\end{array}$ \\
\hline
\end{tabular}

${ }^{\star} \mathrm{p}<0.05 ;{ }^{\star \star} \mathrm{p}<0.01 ;{ }^{\star \star \star} \mathrm{p}<0.001$.

$\mathbf{O}=$ Number of observed deaths. When $\mathrm{O}$ equals zero, the number of expected deaths is given in parentheses.

Relative risk (ratio of SMRs) compared with unexposed group; (a) $=0.99$ (NS); (b) $=1.96$ (NS).

$(\mathrm{SMR}=239, \quad \mathrm{p}<0.05) . \quad$ For cardiovascular diseases, a significant SMR was found only for nonsmokers $(166, p<0.05)$. The excess of stomach cancers for men in byproducts was also only found for non-smokers $(1676, \mathrm{p}<0.001)$.

For lung cancer, non-smokers did not have a significant excess of deaths. For smokers $(\mathrm{SMR}=310, \mathrm{p}<0.001)$ a significant $S M R$ was found for workers on or near the ovens (294, p < 0.01), in the workshops $(581, p<0.001)$, and in the non-exposed workers $(297, \mathrm{p}<0.05)$. Compared with the non-exposed subjects, workers on or near the ovens and those in the workshops had relative risks of 0.99 and 1.96 (NS) respectively. For each exposure group, smokers and subjects having unknown smoking habits had similar SMRs.

The excess of mortality from lung cancer was greater among subjects who worked in plant $A$ than among those in plant $B$ but the difference was not significant. The excess of all cancers was significant only for plant B $(S M R=157, p<0.05)$. Conversely, mortality from cirrhosis of the liver and alcoholism was only increased for plant A (SMR $=190, \mathrm{p}<0.05$; table 6$)$. An interesting question was whether there was an increased risk of lung cancer among the subjects who worked at the beginning of their careers in $A_{\text {old }}$ where the level of exposure to polycyclic aromatic hydrocarbons could

Table 6 Mortality according to plants

\begin{tabular}{|c|c|c|c|c|c|}
\hline \multirow[b]{2}{*}{ Cause of death (ICD-9) } & \multicolumn{2}{|c|}{ Plant $A$} & \multicolumn{2}{|c|}{ Plant $B$} & \multirow{2}{*}{$\frac{\text { Test }}{A v B}$} \\
\hline & $O$ & $S M R$ & $O$ & $S M R$ & \\
\hline All causes & 101 & $134^{\star \star}$ & 81 & $152^{\star \star \star}$ & NS \\
\hline $\begin{array}{l}\text { Cancer, all sites (140-239) } \\
\text { Lung }(162) \\
\text { Upper respiratory tract, alimentary tract }(140-149,161) \\
\text { Oesophagus }(150) \\
\text { Stomach }(151) \\
\text { Bladder (188) } \\
\text { Colon }(153) \\
\text { Rectum }(154)\end{array}$ & $\begin{array}{l}30 \\
16 \\
\frac{1}{2} \\
-1 \\
-\end{array}$ & $\begin{array}{l}115 \\
267 \star \star \star \\
29 \\
(1 \cdot 89) \\
79 \\
(0 \cdot 83) \\
64 \\
(0 \cdot 83)\end{array}$ & $\begin{array}{r}30 \\
9 \\
3 \\
3 \\
2 \\
1 \\
3 \\
1\end{array}$ & $\begin{array}{l}157^{\star} \\
199^{\star} \\
109 \\
210 \\
107 \\
171 \\
274(\mathrm{a}) \\
172\end{array}$ & NS \\
\hline $\begin{array}{l}\text { Cardiovascular diseases }(390-459) \\
\text { Respiratory diseases }(460-519) \\
\text { Cirrhosis of liver, alcoholism }(571.0-3,5) \\
\text { Accidents, other violence }(\text { E800-999) }\end{array}$ & $\begin{array}{r}36 \\
2 \\
10 \\
3\end{array}$ & $\begin{array}{c}129 \\
39 \\
190^{\star} \\
56\end{array}$ & $\begin{array}{r}28 \\
4 \\
3 \\
2\end{array}$ & $\begin{array}{l}139(a) \\
109 \\
71 \\
50\end{array}$ & \\
\hline
\end{tabular}

${ }^{\star} \mathrm{p}<0.05 ;{ }^{\star \star} \mathrm{p}<0.01 ;{ }^{\star \star \star} \mathrm{p}<0.001 ;(\mathrm{a}) \mathrm{p}=0.08$.

$\mathrm{O}=$ Number of observed deaths. When $\mathrm{O}$ equals zero, the number of expected deaths is given in parentheses. 
Table 7 Comparison of mortality from all causes and from lung cancer between subjects having worked and those who did not work in the old generations of plant $A$

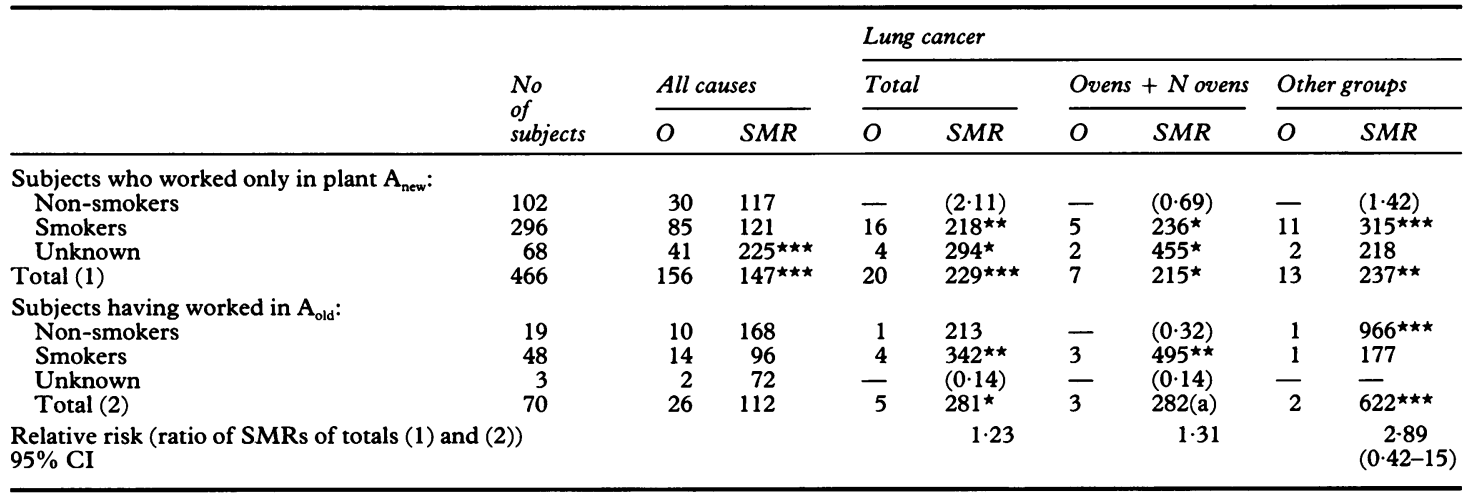

${ }^{\star} \mathrm{p}<0.05 ;{ }^{\star \star} \mathrm{p}<0.01 ;{ }^{\star \star \star} \mathrm{p}<0.001 ;$ (a) $\mathrm{p}=0.06$.

$\mathrm{O}=$ Number of observed deaths. When $\mathrm{O}$ equals zero, the number of expected deaths is given in parentheses.

have been higher than in $A_{\text {new }}$. Table 7 presents the comparative results between the subjects who worked in $A_{\text {old }}$ and the other workers. Unexpectedly the subjects who worked in $A_{\text {old }}$ had a non-significant SMR for all causes (112) whereas those who worked only in $A_{\text {new }}$ had a significant SMR $(147, p<0.001)$. For lung cancer, compared with the subjects who worked only in $A_{\text {new }}$, those who worked in $A_{\text {old }}$ had a relative risk greater than unity $(1.31$ for subjects on or near the ovens, 2.89 for the other subjects, and 1.23 for all groups together) but this was not significant. Compared with the subjects who worked only in $A_{\text {new }}$, those who worked in $A_{\text {old }}$ for more than five years had a relative risk for lung cancer of $1 \cdot 10$ (NS) for men on or near the ovens, 4.31 ( $p=0.06,95 \% \mathrm{CI}=0.59$ 22.00 ) for the other workers, and 1.46 (NS) for all groups together.

\section{Discussion}

The reconstitution of each subject's job history enabled us to define his occupational exposure during his working life. Almost all subjects had one dominant job and the six exposure categories were homogeneous. The total duration of exposure ranged from 18 to 24 years. The non-exposed group also included slightly exposed subjects; they had an average total duration of exposure of 1.2 years. The frequency of unknown causes of death $(11.0 \%)$ was close to that of the French male population used as the standard population.

Smoking habits were determined for $86.8 \%$ of the subjects. Because of small numbers, we did not distinguish between smokers and ex-smokers. This is a weakness that stems from the fact that the frequency of subjects having unknown smoking was significantly higher in dead subjects than in survivors
$(23.6 \% v 7.9 \%, p<0.001)$. Thus the mortality of non-smokers and smokers may be underestimated. This bias may conceal in part the effect of occupational exposures.

The choice of the standard population was important. We were tempted to choose the population of Lorraine $\left(1.16\right.$ million men in $\left.1975^{41}\right)$ or, better, that of the administrative division of Moselle $\left(0.51\right.$ million men in $\left.1975^{41}\right)$ because the mortality from lung cancer is higher in these regions compared with that of France (SMR 140 for Lorraine and 159 for Moselle in the 1971-78 period ${ }^{41}$ ). Unfortunately, the statistics for these areas are not available for the years 1963-74. Pursuing this logic, however, the most appropriate standard population was that of the coalmine district as this region was exposed to fairly high atmospheric pollution produced by the industrial platforms. ${ }^{42}$ Unexpectedly, this area has, for 1980-1984, an SMR for lung cancer equal to that of all Lorraine ${ }^{43}$ but these figures concern a period different from that of the present study.

Although the phenomenon of lower mortality in working populations, ${ }^{328}$ the healthy worker effect, is frequently reported, our study showed a total mortality higher than expected $(S M R=141$, $\mathrm{p}<0.001)$. This excess mortality was found among subjects who worked near the ovens $(145, \mathrm{p}<0.01)$ and in byproducts $(176, \mathrm{p}<0.05)$, but also among non-exposed workers $(S M R=163, p<0.001)$. In the previous study ${ }^{1}$ the excess of overall mortality was small $(S M R=107)$ and non-significant. The increase in the death rate found in the five year interval allowed us to consider that the deterioration in health of the study retirees compared with a normal population was accelerated with age. Increasing mortality was found for several causes, however, (especially for cardiovascular diseases $(S M R=133$, $\mathrm{p}<0.05)$ ) whereas the SMR for lung cancer did not 
vary (251 $v 238)$. This seemed to show that in the retirees the competing risk may change with age. It is interesting that the age at death varied slightly with the cause: 63.3 (SD 1.4) years for lung cancer, 63.5 (SD 1.0) years for other cancers, 66.4 (SD 0.8 ) for cardiovascular diseases, and 64.5 (SD 1.1) for other causes; the difference between overall cancer and cardiovascular diseases was significant $(\mathbf{p}<0.05)$. Consequently, the age of the subjects may explain in part the differences between these results and those of other investigators.

An excess of mortality from lung cancer was found for all exposure groups except for those working underground; but probably due to small numbers, it was significant only for subjects who worked near the ovens ( $S M R=252, p<0.01)$, in the workshops $(S M R=433, p<0.001)$ and in non-exposed workers $(S M R=228, p<0.05)$. This investigation confirmed that the excess of deaths from lung cancer was slightly higher in plant $A$ than in plant $B$, but the difference was not significant. Compared with the subjects who worked only in $A_{\text {new }}$, those who worked in $A_{\text {old }}$ had a relative risk for lung cancer higher than unity but it was not significant. Regarding mortality in Lorraine, the excess of lung cancer could not be explained solely by the regional factor.

The proportion of smokers in the study population ( $74 \%$; subjects with unknown smoking habits excluded) was slightly lower than that in the French male population (about $80 \%){ }^{44}$ A question of interest was whether this difference could explain the increased value of SMR for lung cancer. Using the method proposed by Axelson and Steenland ${ }^{45}$ and suggested by Moulin, ${ }^{46}$ and assuming a risk for lung cancer equal to unity for non-smokers and up to 10 for smokers (these values were close to those habitually exposed), ${ }^{47}$ the relative risk of the study population was close to unity compared with the French male population. On the other hand, in the present study, mortality from upper respiratory and alimentary tract cancer, usually attributed to smoking, was low $(S M R=64)$ even for smokers $(\mathrm{SMR}=78)$.

A significant excess of deaths from stomach cancer was found for workers in byproducts $(S M R=538$, $\mathrm{p}<0.01$ ), from colon cancer for those near the ovens $(S M R=381, p<0.05)$, and from cirrhosis of the liver and alcoholism for those underground $(S M R=460, p<0.01)$, but, due to small numbers, these figures were not robust. The increased risk for cardiovascular diseases was significant for workers near the ovens $(S M R=155, p<0.05)$ but also for non-exposed subjects $(S M R=179, p<0.01)$.

The increased values of the SMRs for all causes of death, lung cancer, and cardiovascular diseases could be explained in part by the fact that the study subjects were unskilled workmen. Indeed, they had a life expectancy lower than that of the French male population. ${ }^{48}$ For the same number of deaths, the value of the SMR is higher when the deaths intervene earlier. This factor could not totally explain the excess mortality, however, because the value of the SMR varied greatly with the cause of death, and the highest value was found for lung cancer.

Comparison with the results of other investigations is difficult because of the differences in the choice of the study population and of the standard population, the nature and the level of the hazards, which could change with time, ${ }^{7}$ the healthy worker effect, the socioeconomic state of the workers, the method of data collection, etc. The concentration of polycyclic aromatic hydrocarbons was higher for the American coke ovens than for those in Europe. ${ }^{29}$ Also, there were racial differences among the workers. For example, the American coke ovens were mainly operated by non-white workers. On the other hand, there were also further hazards such as heat, coal dust, and work conditions. In this investigation, the study subjects were exposed, moreover to atmospheric pollution at their workplace and sometimes at their home, which was often near the Carling or Marienau industrial platforms. ${ }^{42}$

Kennaway and Kennaway ${ }^{13}$ found, for coke oven workers in England and Wales from 1921 to 1938, a death rate from lung cancer $2 \cdot 8$ times and from laryngeal cancer $2 \cdot 1$ times higher than expected in reference to the general population. In 1972 Doll et $a l^{4}$ noted that among 4700 coal gasification workers in Great Britain, the subjects with the highest exposure had a risk higher than the general population for bladder cancer, scrotal and skin cancer, lung cancer, and bronchitis. Davies ${ }^{3}$ stated that among 610 coke plant workers overall mortality was lower than expected and no excess of deaths from cancer was found. In 1977, Jacobsen and Hurley ${ }^{10}$ found a death rate of lung cancer $29 \%$ higher than expected among 4836 men in the British industries of coal carbonisation and tar distillation. This difference was significant but there were important variations between various plants. Recently, in a study on 6673 coke workers employed in 1967 in 27 plants throughout Britain, Hurley $e t a l^{7}$ found an excess of mortality from lung cancer that was related to estimates of exposure of workers to concentrations of benzene soluble materials up to 1967 . They reported a significant association between stomach cancer and the time worked in byproducts. Thus this result supports our finding. Recently, these authors ${ }^{8}$ reported that excess of mortality from leukaemia had not been identified among coke oven and other coal product workers. In our study, two deaths occurred from this cause.

In 1991, Swaen et al ${ }^{29}$ reported the results of a mortality study in the Netherlands of 5639 subjects who had worked in a coke plant for more than six months between 1945 and 1969 compared with 5740 who had worked in a nitrogen fixation plant during 
the same periud. These authors found a death rate of lung cancer and of non-malignant respiratory diseases significantly higher than expected among the coke oven workers, but the workers in byproducts had a mortality similar to that expected. They also found the highest overall mortality for subjects who worked in the oldest plant (started in 1918). In our investigation, an increased risk for lung cancer was shown in the former generations of the plant A, although it was not significant.

It was interesting to enlarge the comparison to an investigation by Pham et al ${ }^{49}$ on mortality in 13801 iron miners in the same Lorraine area (this eliminates the ethnic problem) between 1982 and 1986. These authors found that among smokers, in reference to the French male population, a proportionate mortality ratio (PMR) for lung cancer was linked to the duration of underground work (2.04 for 1-19 years, 2.32 for $20-29$, and 3.05 for $\geqslant 30$ years), but the differences were not significant. They reported a significant PMR (2.31) for stomach cancer, but this did not vary significantly with the duration of underground work. As in our investigation, these authors also found a mortality from upper respiratory and alimentary tract cancer lower than expected for smokers.

In the United States, Lloyd ${ }^{15}$ reported a mortality from lung cancer 2.5 times higher for workers on ovens of the Alleghany County coke ovens plants, than for other steelworkers. Redmond $e t$ al ${ }^{17}$ showed that the death rate from lung cancer was linked to duration and amount of exposure while working on the ovens: the death rates of lung cancer were respectively 2.5 and 3.5 times higher than expected for men employed on the ovens and for those at the work place for more than five years. Also, the relative risk for lung cancer was $2 \cdot 1$ times higher for men working only near the ovens, 3.2 for those on and near, and 6.9 for those on the ovens. In 1979, Redmond et al ${ }^{18}$ reported that non-white coke oven workers had a risk for lung cancer twice higher than other steelworkers, and that the risk was eight times higher for men working on the ovens.

In Japan, Kawai et $a l^{12}$ reported a higher mortality from lung cancer than expected among workers of a coal gasification plant. Sakabe et al ${ }^{28}$ found, for 4655 retired employees from 36 steelworks, a mortality from all cancer sites and from lung cancer similar to that of the general population; none the less, among workers from coke oven plants, mortality from lung cancer was twice as high as expected.

Finally, despite several unknown factors (healthy worker effect, selection at hiring, etc), the results obtained in this study seem to show that the role of occupational hazards could not be excluded. The effect of hazards in the old generations of plant could be clarified if the cohort were followed up for a few more years.

\section{Conclusion}

This study showed a significant excess of deaths from all causes, all cancers, and cardiovascular diseases, whereas for lung cancer the SMR did not change compared with that in the previous study. A significant excess mortality was found for all causes, lung cancer, colon cancer, and cardiovascular diseases for subjects who worked near the ovens. This investigation shows that the ovens were not the only exposed work places. A significant excess mortality was also found for subjects who worked in byproducts for all causes and stomach cancer, for those in the workshops for lung cancer, and for those underground for cirrhosis of the liver and alcoholism. An excess of deaths from all causes, lung cancer, and cardiovascular diseases was, however, also found, for non-exposed or slightly exposed subjects. The fact that on the whole the mortality of various exposed groups was similar to that of non-exposed or slightly exposed workers may be explained in part by the selection at hiring and the healthy worker effect. As an increased risk of lung cancer was noted among subjects who worked in the old generations of plant compared with the other workers (although the relative risk was not significant) we concluded that the role of occupational hazards could not be excluded. A directive of 28 July 1990 of the Council of the European Community ${ }^{50}$ gives measures to fight such occupational hazards.

We thank all the physicians, notably those of Mines Social Security (SSM) and "Hospitalor", and the staff of the coke oven plants and of Lorraine coalmining occupational medicine for their helpful cooperation. We also thank F Biller for the statistical calculations.

1 Bertrand JP, Chau N, Patris A, et al. Mortality due to respiratory cancers in the coke oven plants of the Lorraine coalmining industry (Houillères du Bassin de Lorraine). $\mathrm{Br} J$ Ind Med 1987;44:559-65.

2 Bross IDJ, Viadana E, Houten L. Occupational cancer in men exposed to dust and other environmental hazards. Arch Environ Health 1978;33:300-7.

3 Davies GM. A mortality study of coke oven workers in two south Wales integrated steelworks. Br J Ind Med 1977;34:291-7.

4 Doll R, Vessey MP, Beasley RWR, et al. Mortality of gasworkers. Final report of a prospective study. $\mathrm{Br} J$ Ind Med 1972;29:394-406.

5 Falk HL, Jurgelski WJ. Health effect of coal mining and combustions: carcinogens and cofactors. Environ Health Perspect 1979;33:203-26.

6 Hurley JF, Archibald R, Collings PL, Fanning DM, Jacobsen $M$, Steele RC. The mortality of coke workers in Britain. Am J Ind Med 1983;4:691-704.

7 Hurley JF, Cherrie JW, Maclaren WM. The mortality of coke workers in Britain: a 20-year follow-up. Edinburgh: Institute of Medicine, 1991. (IOM report TM/91/01.)

8 Hurley JF, Cherrie JW, Maclaren WM. Exposure to benzene and mortality from leukaemia: results from coke oven and other coal product workers. Br J Ind Med 1991;48:502-3.

9 International Agency for Research on Cancer. Monographs on the evaluation of the carcinogenic risk of chemicals to humans. Polynuclear aromatic compounds. Vol 34, part 3, Coke production. Lyon: IARC 1984.

10 Jacobsen M, Hurley JF. Mortality at British factories engaged in 
coal carbonisation and tar distillation. Edinburgh: Institute of Occupational Medicine, 1977.

11 James WRL. Primary lung cancer in South Wales coal-workers with pneumoconiosis. Br J Ind Med 1955;12:87-91.

12 Kawai M, Amamoto A, Harada K. Epidemiologic study of occupational lung cancer. Arch Environ Health 1967;14: $859-67$.

13 Kennaway E, Kennaway N. A further study of the incidence of cancer of the lung and larynx. BrJ Cancer 1947;1:260-5.

14 Kennaway E, Kennaway N. The incidence of cancer of the lung in coalminers in England and Wales. Br J Cancer 1953;7: $10-18$.

15 Lloyd JW. Long-term mortality study of steelworkers: respiratory cancers in coke plant workers, J Occup Med 1971;13:53-68.

16 Lloyd JW. Lung cancer mortality in Alleghany County coke-plant workers. (thesis). Pittsburgh, PA: University of Pittsburgh, 1966.

17 Redmond CK, Ciocco A, Lloyd JW, Rusch HW. Long-term mortality study of steelworkers: mortality from malignant neoplasms among coke oven workers. J Occup Med 1972; 14:621-9.

18 Redmond CK, Wienand HS, Rockette HE, Sass R, Weinberg G. Long term mortality experience of steelworkers. Report prepared by the University of Pittsburgh, Pennsylvania, for NIOSH. Cincinnati, Ohio: 1979. (Contract No HSM-99-71-32)

19 Mazumdar S, Redmond CK, Sollecito W, Sussman N. An epidemiological study of exposure to coal tar pitch volatiles among coke oven workers. J Air Pollut Control Assoc 1975; 25:382-9.

20 Meijers JMM, Swaen GMH, Slangen JJM and Van Vliet C. Lung cancer among Dutch coal miners: a case-control study. Am J Ind Med 1988;14:597-604.

21 Mooney F. Carcinoma of the lung in Lancashire coalminers. Thorax 1979;34:826-7.

22 Moulin JJ, Mur JM, Wild P, Demonchy A, Eloy E, Jeannot A. Etude epidémiologique de mortalité parmi les salariés d'une usine de distillation des goudrons de houille. Revue d'epidémiologie et de santé publique 1988;36:99-107.

23 National Institute for Occupational Safety and Health. Criteria for a recommended standard occupational exposure to coal tar products. Cincinnati: NIOSH, 1977 (DHEW pub No 78-107).

24 Reid DD, Buck C. Cancer in coking plant workers. Br J Ind Med 1965;13:265-72.

25 Robertson JMD, Ingalls TH. A mortality study of carbon black workers in the United States from 1935 to 1974. Arch Environ Health 1980;35:181-6.

26 Rockette HE. Cause specific mortality of coalminers. J Occup Med 1977;19:795-808.

27 Rooke GB, Ward FG, Dempsey A, Dowler JB, Whitaker CJ. Carcinoma of the lung in Lancashire coalminers. Thorax 1979;34:229-33.

28 Sakabe H, Tsuchiya $\mathrm{K}$, Takerura N, et al. Lung cancer among coke oven workers. Report to labour standard bureau, Ministry of Labour, Japan. Ind Health 1975;13:57-68.

29 Swaen GMH, Slangen JJM, Volovics A, Hayes RB, Scheffers T, Sturmans F. Mortality of coke plant workers in the Netherlands. Br J Ind Med 1991;48:130-5.

30 Swaen GMH, Aerdts CWHM, Slangen JJM. Gastric cancer in coalminers: final report. $B r J$ Ind Med 1987;44:777-9.

31 Mayer L, Chau N, Bertrand JP, et al. Morbidity in retired coke oven plant workers. Am J Ind Med 1993 (in press).
32 Chau N, Bertrand JP, Guenzi M, Mayer L, Téculescu D, Mur $\mathrm{JM}$, et al. Lung function in retired coke oven plant workers. $\mathrm{Br}$ $J$ Ind Med 1992;49:316-25.

33 Organisation Mondiale de la Santé (OMS). Classification internationale des maladies. 9ème revision. Genève: OMS, vol 1, 1975; vol 2, 1977.

34 Armitage P, Berry G. Statistical methods in medical research. Oxford: Blackwell Scientific Publications, 1987.

35 Grémy F, Salmon D. Bases statistiques. Paris: Dunod, 1969.

36 Breslow NE, Day NE. Statistical methods in cancer research. Vol II. The design and analysis of cohort studies. Lyon: International Agency for Research in Cancer, 1987. (IARC sci publ IVO 82)

37 Mantel N, Haenzel W. Statistical aspects of the analysis of data from retrospective studies of disease. J Natl Cancer Inst 1959;22:719-48.

38 Peto P, Pike MC, Armitage P, et al. Design and analysis of randomized clinical trials requiring prolonged observation of each patient. Br J Cancer 1977;35:1-39.

39 INSERM. Statistique des causes médicales de décès, Vol 1. Résultats France entière. 1963-1987. Paris: INSERM, 1987.

40 Chau N, Patris A, Martin J, Kohler F, Lambert JP. Conception d'un logiciel de traitement et d'interrogations statistiques de données LQGIST. Int J Biomed Comput 1985;16:95-118.

41 Rezvani A, Doyon F, Flamant R. Atlas de la mortalité par cancer en France (1971-1978). Paris: Les Editions INSERM, 1986.

42 Patris A, Chau N, Téculescu D, Marchand F, Aubry C, Pham $\mathrm{QT}$, et al. La pollution atmosphérique dans la plateforme industrielle de Carling en 1984. Pollution Atmosphérique 1987;116:373-81.

43 Briançon S, Castel O, Hilico C, Schléret Y. Atlas de la mortalité en Lorraine. Observatoire régional de la santé et des affaires sociales en Lorraine, Nancy: INSEE, 1989.

44 Service des Statistiques, des Etudes et des Systèmes d'Information (SESI). Les consommateurs dê boissons alcooliques et de tabac. Enquête sur la santé et les soins médicaux 1980-1981. Solidarité Santé. Cahiers statistiques 1984; No 1.

45 Axelson $\mathrm{O}$, Steenland $\mathrm{K}$. Indirect methods of assessing the effects of tobacco use in occupational studies. Am J Ind Med 1988;13:105-8.

46 Moulin JJ. Les études épidémiologiques de cohortes industrielles: comment prendre en compte le tabagisme? Arch Mal Prof 1991;52:319-26.

47 Surgeon General. The health consequences of smoking. Cancer. Washington: US Department of Health and Human Services, 1982.

48 Desplanques G. La mortalité des adultes suivant le milieu social. 1955-1971. Paris: INSEE, 1976. (No 195 des collections de l'INSEE.)

49 Pham QT, Chau N, Patris A, Trombert B, Henquel JC, Geny $\mathrm{M}$, et al. Prospective mortality study among iron miners. Cancer Detection and Prevention. 1991;15:449-54.

50 Conseil des Communautés européennes. Directive du Conseil du 28 juin 1990 concernant la protection des travailleurs contre les risques liés à l'exposition à des agents cancérigènes au travail (sixième directive particulière au sens de l'article 16 paragraphel de la directive 89/391/CEE). Brussels: Journal officiel des Communautés européennes No L 196/1 du 26 juillet 1990.

Accepted 27 April 1992 